\title{
R \& Ds for 21st Century Photonic Network in Japan
}

\author{
Ken-ichi Kitayama \\ Osaka University \\ 2-1 Yamadaoka, Suita, Osaka 565-0871, JAPAN \\ kitayama@comm.eng.osaka-u.ac.jp
}

\begin{abstract}
Government-funded R\&D initiatives have been playing crucial roles for developing photonic networks. The $\mathrm{R} \& \mathrm{D}$ project is typically carried out for a relatively long period of five-years, and the goal is set so high that no single private sector can afford the risk. In this paper, challenging governmentfunded R\&D initiatives of photonic networks in Japan is introduced. They have been launched in 2006, which aims to establish the photonic platform to provide abundant bandwidth on demand, at any time and from anywhere, to promote bandwidth-rich networked applications. In addition, other governmentfunded R\&D activities relevant to the photonic networks in Japan is also presented, including photonic devices and subsystems and large-scale optical interconnect for ultra-high performance computing.
\end{abstract}

Keywords: R\&D initiative, photonic network, optical communication, network testbed, government fund, Japan

\section{Introduction}

Government R\&D initiatives have been playing crucial roles to evolve voice-oriented legacy telecommunication networks to IP-based NG networks [1]. The R\&D programs are characterized by participants, the period, and the budget size as well as the goals. A consortium is first formed consisting of both academic and industrial partners, the $R \& D$ is typically carried out during a relatively long period of four- or five-years, which is financially well supported, and goals are set so high that no single private sector can afford the risk.

In this paper, challenging government-funded R\&D initiatives of photonic networks in Japan is introduced. They have been launched in 2006, which aims to establish the photonic platform to provide abundant bandwidth on demand, at any time and from anywhere, to promote bandwidth-rich networked applications. In addition, other government-funded R\&D activities relevant to the photonic networks in Japan is also presented, including photonic devices and subsystems and large-scale optical interconnect for ultra-high performance computing. 


\section{Photonic Network R\&D Initiatives for Builiding Photonic Platform}

\subsection{Overview}

Four R\&D projects of photonic networks have been launched in 2006 under the financial support of an agency of the Japanese government, the National Institute of Information and Communications Technology (NICT). The outline is summarized in Table 1.

Table 1. R\&D projects supported by NICT

\begin{tabular}{|c|c|c|}
\hline Project & Period & Participants \\
\hline $\begin{array}{l}\text { Photonic node with } \\
\text { multiple granularity } \\
\text { switching capability }\end{array}$ & 2005-2009 & $\begin{array}{l}\text { NTT Comm., Fujitsu, NTT, } \\
\text { Nagoya Univ. }\end{array}$ \\
\hline$\lambda$ Access & 2006-2010 & $\begin{array}{l}\text { NTT, NEC, NTT Com., } \\
\text { Mitsubishi Electric, Hitachi, } \\
\text { KDDI Labs., Univ.Tokyo, } \\
\text { Keio Univ. }\end{array}$ \\
\hline$\lambda$ Utility & 2006-2010 & $\begin{array}{l}\text { NEC, Fujitsu, Mitsubishi } \\
\text { Electric, Oki Electric, Osaka } \\
\text { Univ. }\end{array}$ \\
\hline $\begin{array}{l}\text { Optical RAM for } \\
\text { all-optical packet } \\
\text { switching }\end{array}$ & 2006-2010 & $\begin{array}{l}\text { NTT, NEC, Osaka Univ., } \\
\text { Kyushu Univ. }\end{array}$ \\
\hline
\end{tabular}

\subsection{Photonic Node with Multi-granularity Switching Capability}

The objective of the project is to develop fundamental technologies for 100 Tera-bps class photonic nodes with multiple granularity switching capability and for the design and control of backbone networks with Peta-bps class throughput utilizing the multigranularity nodes. As shown in Fig.1 the reesearch themes are the followings;

- The architecture of large-scale optical switches with several nano-second switching time for handling optical burst data. Materials, devices, module fabrication and control techniques for large-scale high-speed optical switches.

- The architecture of a multi-granularity photonic node that can handle optical bursts to/from waveband paths. Key technologies for realizing the node hardware such as multi-wavelength light sources, optical virtual concatenation, waveband conversion, and stable optical modulation/demodulation with highly spectral efficiency. 
- The architecture and design of a photonic network that employs hierarchical optical paths. The control technology of combined optical switches with different switching granularities.

\section{Tbps-Class photonic node with multiple granularity switching capability -Ultra-fast Switching technology with nsec order for optical burst handling. \\ - Node technology with switching granularity up to waveband for throughput expansion. -Architecture and networking of Photonic NW with multiple switching granularity.}

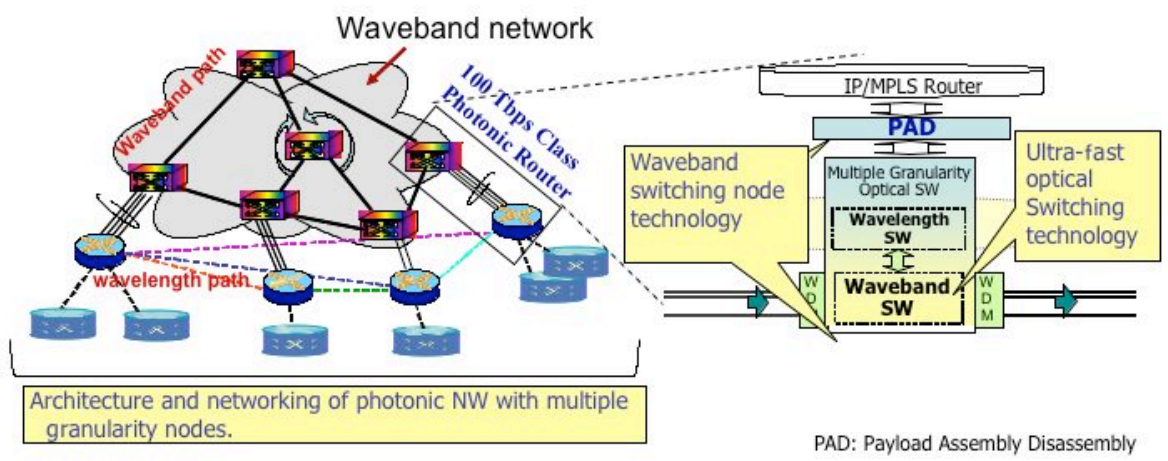

Fig. 1 High performance photonic node with multi-granularity switching capability

\section{$2.3 \quad \lambda$ Access}

Multiple-lambda optical networks envision virtual Terabit LAN in geographical scale, and two of its key technical challenges are to provide user network interface (UNI) with scalability and controllability. The "Lambda Access" is a new five-year project funded for these challenges in Japan by NICT (Nation Institute of Information and Communications Technology), that focuses on network access at 100Gbps and beyond with single or multiple lambda interfaces in cooperation with the related project "Lambda Utility". As shown in Fig. 2, the Lambda Access Project includes the following two themes;

- WDM Seamless Access Technology

This theme aims at the establishment of Terabit LAN network interfacing technology that allows an end-user to access network with multiple lambdas at 100Gbps and more. It includes user-controlled link bonding for scalable access, new-generation MAC with Mega-Byte-size frames, and network access protocols for seamless userside controllability.

- $\quad$ Frame-multiplexed Ultra High Speed Access Technology 
This theme aims at the establishment of Ethernet frame aggregator and its uplink single-lambda $100 \mathrm{Gbps}$ interface. It includes statistic Ethernet frame aggregation at $100 \mathrm{G}$ bps and beyond, 100Gbps single-lambda modulation/demodulation techniques as well as its physical layer implementation, and frame-base protocols for end-to-end OAM.

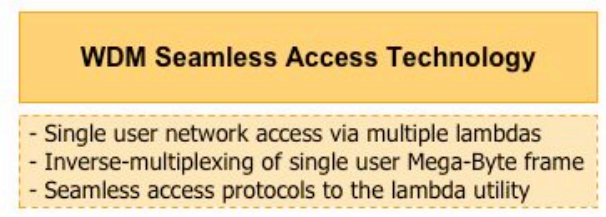

Inverse-multiplexing of single user Mega-Byte frame
Seamless access protocols to the lambda utility

\section{Frame-Multiplexed Ultra High Speed Access Technology}

Multiple user network access via single lambda

Statistic aggregation of multiple user frames

- End-end frame-base seamless OAM protocols

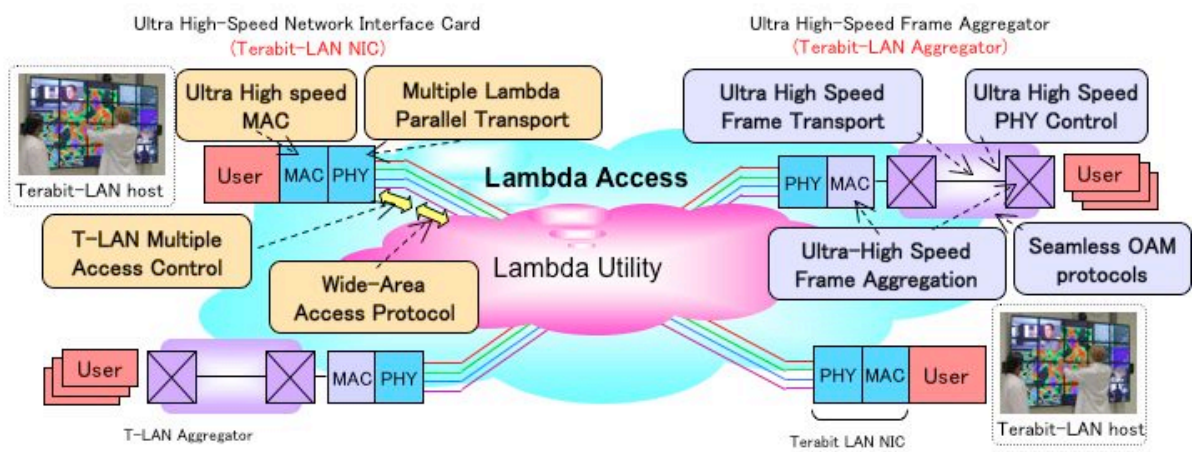

Fig. 2. Outline of $\lambda$ Access project

\section{$2.4 \lambda$ Utitity}

The Lambda Utility Project cooperating with the Lambda Access Project performs technical development which realizes the terabit-class LAN environment over wide area networks. The goal of these projects is to provide each end-user stress-free 100Gbps-class bidirectional communication with various services, such as a super high definition video conference, GRID computing, and so on, as shown in Fig. 3. The Lambda Utility Project, focusing on technologies in a wide area network, includes the following three themes;

- $\quad$ Borderless optical path control and management technology

This theme aims at the establishment of photonic service gateway technology which utilizes the optical path over multiple wide area networks with scalability of more than 1000 nodes.

- Highly-spectral-efficient transmission link technology 
This theme aims at the establishment of optical link technology at 100Gbps and beyond. It includes the multi-level modulation and demodulation technology for pursuing high spectral efficiency. The high coding gain forward error correction code is also included in this theme to cope with the higher signal to noise ratio requirement for the multi-level modulation format.

- $\quad$ Modulation-format-independent all-optical 3R technology

This theme aims at the establishment of all-optical 3R technology which is capable for both phase modulation format signal and intensity modulation format signal at 100Gbps and beyond.

It is noted that the Lambda Utility Project cooperates with the Lambda Access Project, and those two projects will conduct the interoperability experiment for realizing a terabit-class LAN over wide area networks.

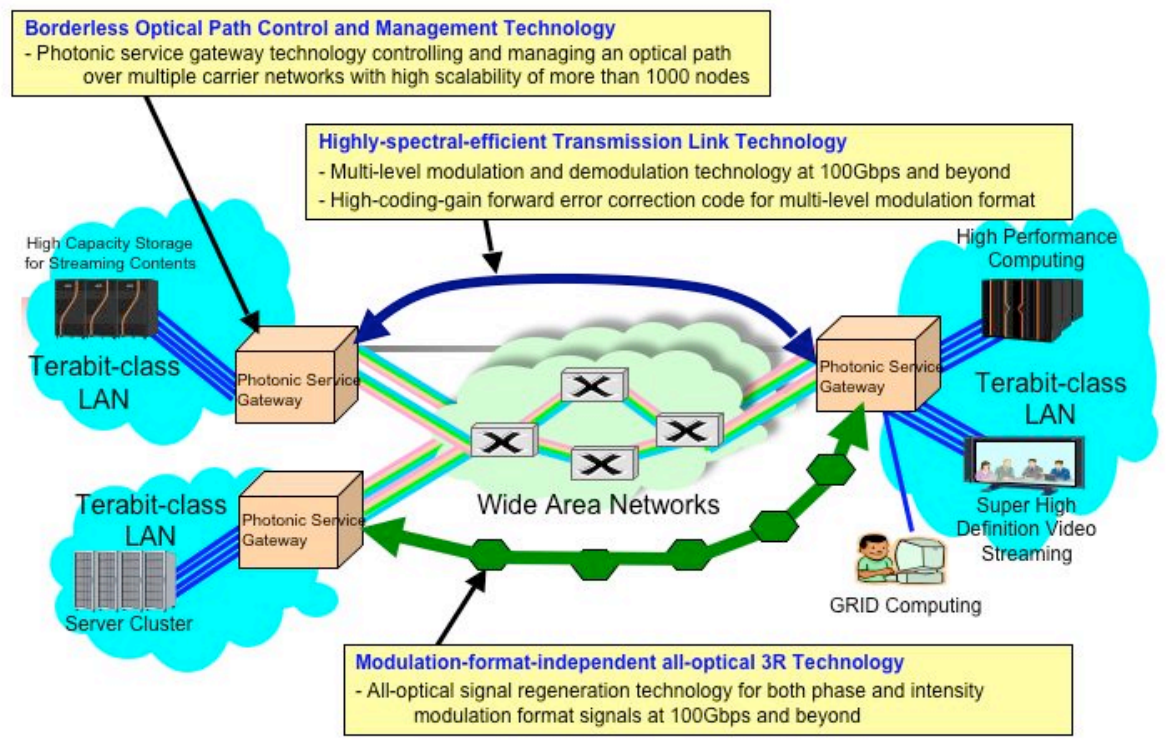

Fig. 3. Outline of $\lambda$ Utility project

\subsection{Optical RAM for All-optical Packet Switchings}

This project aims at developing basic technology for all-optical packet switch. A main focus is on optical random access memory (RAM). Three research topics of this project includes are the following;

- Nano-structured optical devices for a bit memory based upon ultrafast bistability. 
- $\quad$ Basic technology for the fabrication process of nano-structured optical devices and their materials.

- Optical interface and subsystem for optical RAM focusing on the optical addressing to the bit memory and the serial-to-parallel conversion of the bitserial optical packet.

- $\quad$ Architecture of all-optical packet switch and buffer scheduling as well as its transmission control protocol, preferably adopted for small buffering capacity.

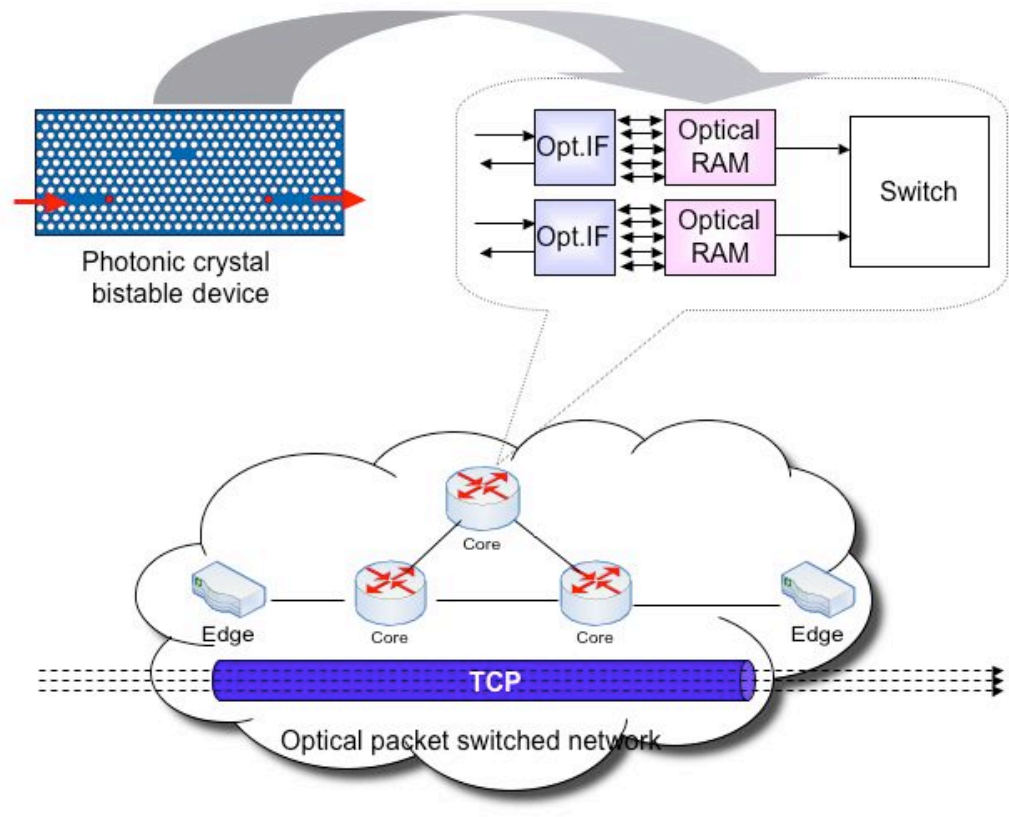

Fig. 4. Outline of optical RAM project.

\subsection{Network Testbeds}

The configuration of the Japan Gigabit Netowrk (JGN) II [2] and the experimental network is shown in Fig.5. JGNII's L2 service used for the GMPLS control layer (CPlane) made it possible to achieve interoperation among Keihanna Center, Otemachi, and Koganei. The C-Plane is used to enable GMPLS nodes to exchange control protocols. For the transport layer (D-Plane), on the other hand, JGNII's STM-64 (standard for $10 \mathrm{Gbps}$ synchronous transmission) service was used. STM-64 was employed under constant connection, with the GMPLS nodes at both ends controlled as a lambda path (LSC-LSP). A multi-layer control experiment was conducted by controlling the STM16 (standard for 2.5-Gbps synchronous transmission) path, with GMPLS as a TDM path (TDM-LSP) and connecting to the MPLS network (MPLSLSP). In the meantime, settings for a virtually wider control network (total extension 
distance for signaling: $1,320 \mathrm{~km}$ ) ere attempted by dividing the control layer in two and causing signals to perform a round trip between Koganei and Keihanna. As for the multi-vendor environment, verification was carried out by combining different items of equipment from nine different vendors and generating several types of paths.

JGNII also is provides with optical testbeds both in Tokyo and Keihana. Field experiments of data-granularity-flexible reconfigurable OADM with wavelengthpacket-selective switch [3] and optical quantum cryptosystem [4] have been conducted.

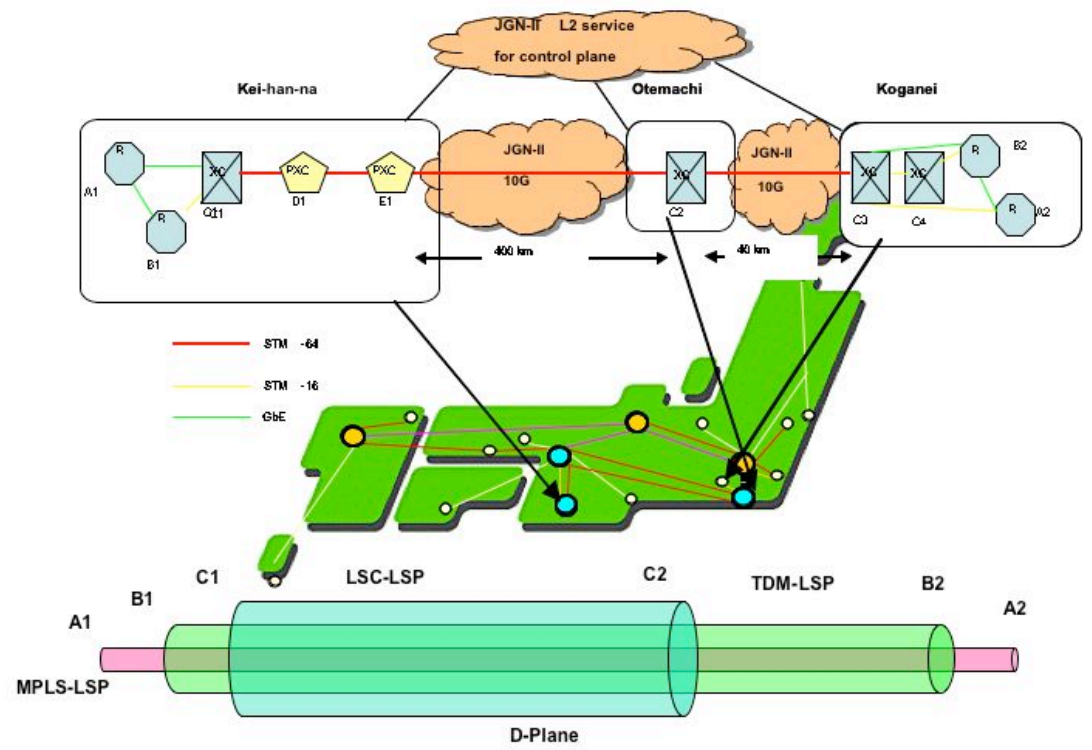

Fig. 5. GMPLS nation-wide interoperability test on Japan Gigabit Netowrk (JGN) II

\section{Photonic Devices and Subsystems R\&D Initiative}

There has been a five-year long R\&D project (2002-2006) for devices and subsystems, "Photonic Network Technology Development Project," funded by New Energy and Industrial Technology Development Organization (NEDO). The project goals are to develop advanced photonic components and demonstrate the feasibility of optical label-switched networking. The consortium was organized by Optoelectronics Industry and Technology Development Association (IOTDA). In the consortium University of Tokyo, KDDI Laboratory, Fujitsu, Mitsubishi Electric, Furukawa Electric, Hitachi, and Hitachi Cable in the consortium has demonstrated 40/10Gbps bit-rate transparent optical burst switching (OBS) [5]. In the experiment the bit-rate transparent switching and wavelength contention resolution as well as the optical 
label processing were performed. Using a PLZT-based 5x5 optical matrix switch, a 2 wavelength, $2 \times 2$ node was developed. A monolithically integrated MZI-SOA wavelength converter was optimized for single-arm $40 \mathrm{Gbps}$ operation, and was used for contention resolution in a shared loop-back configuration. A fast tunable DBR laser diode was used to switch wavelengths within a $\mu$ s. The wavelength converter supported both 10 and 40Gbps PRBS $2^{7}-1$ payloads under same bias conditions to simulate a network supporting multiple bit-rate payloads. Error-free (BER $<10^{-9}$ ) wavelength conversion and switching was achieved for less than $1 \mathrm{~dB}$ and $4.8 \mathrm{~dB}$ power penalty for 10 and $40 \mathrm{Gbps}$ payloads, respectively. The results underline the feasibility of bit-rate transparent fast optical networking.

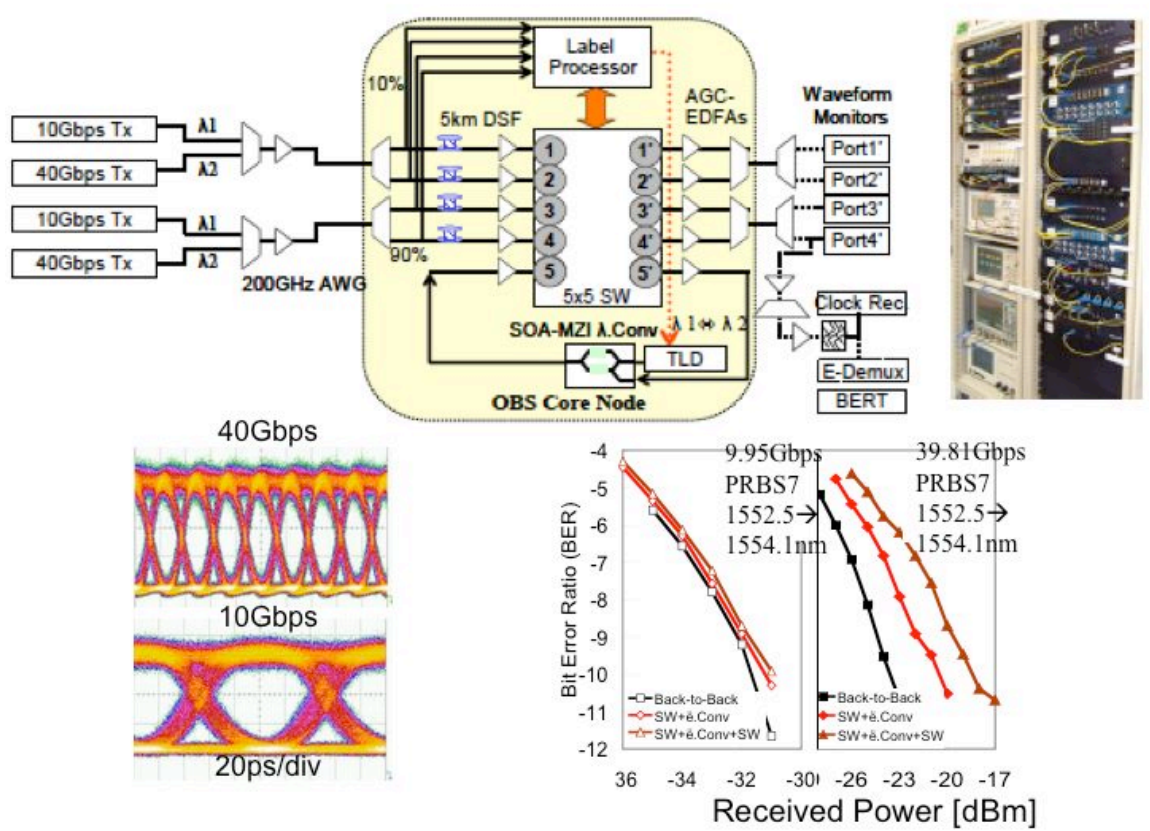

Fig. 6. Experimental Setup for Bit-rate Independent Switching and photo of optical router prototype, in addition to the BER measurement results for contention resolution.

\section{Large-scale optical interconnect for UHPC}

Peta-scale system interconnect (PSI) project [6] is one of the national projects on elemental technologies for ultra-high-performance computing systems, funded by the Ministry of Eduction, Culture, Sport, Science and Technology (MEXT). It is a sixyear long project in the period of 2006-2011. The project works intensively on WDM optical packet interconnect to enables peta-scale supercomputers with over 10,000 compute-nodes. For example, 1 peta-flops system consists of 100 computing node groups, where each group has 200 CPUs and 10 Tflops capability, and the groups are interconnected by $1 \mathrm{~T}$ Byte/s class interconnection link. Thus, 100-Tbps class (100 port $\mathrm{x}$ Tbps class link) interconnection is required for the peta-flops system. The data 
granularity ranges from 64-bytes-length short data to several mega-byte bulk data, and the latency with in microsecond-order latency are necessary to obtain the high simulation speed. One of the big issues facing realization of such interconnection systems is the huge amount of interconnecting elements, especially switches, cables, and OE/EO modules. In the proposed network archtecture [7], an electric leaf switch is used for the switching of the intra-rack signals and the aggregation of the inter-rack signals by using packet queues for high bandwidth data interconnection. The aggregated signals are converted to optical packet signals and interconnected by the optical spine switch among computing node groups under the control of the arbiter. WDM packet switching is used to increase the bandwidth per switching port and reduce the number of OE/EO modules and cables. Broadcast and select type of optical switch with multi-gate semiconductor optical amplifier (SOA) switch to realize both large port counts ( 256port) and nano-second order switching has been developed by Fujitsu. The number of switches, cables and the power consumption are significantly reduced by WDM optical packet switching comparing with conventional all-electric swithing system because the characteristics of the optical switch are independent of the signal bit rate.

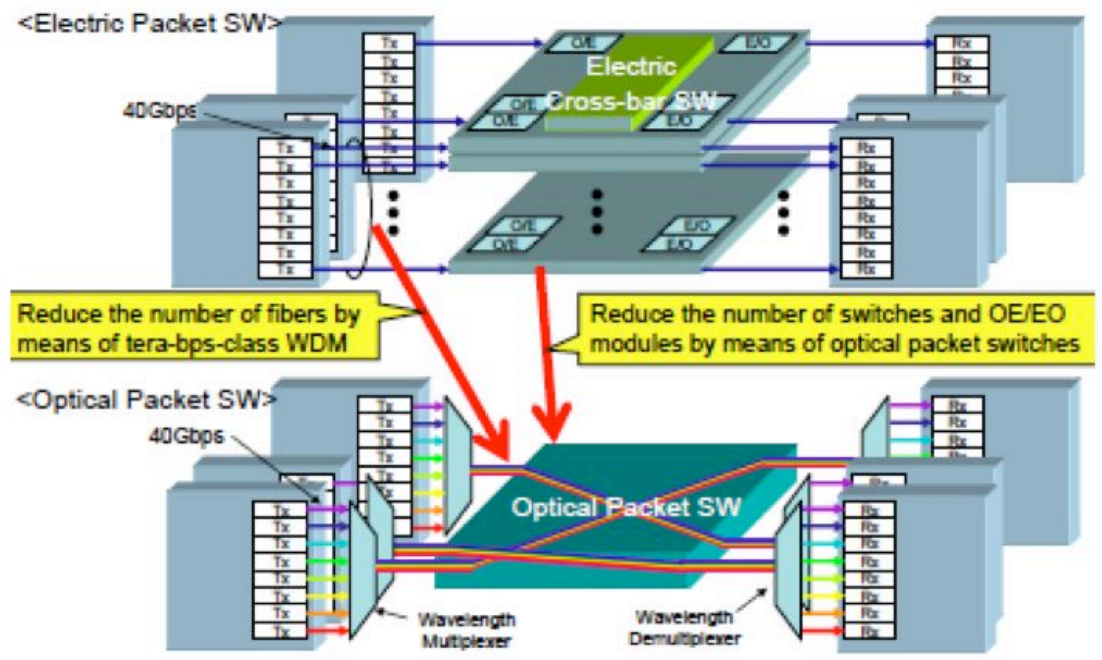

Fig. 7. WDM packet switched interconnect for UHPC.

\section{Conclusion}

Newly launched government-funded R\&D initiatives of photonic networks in Japan has been presented. They aim at establishing the photonic platform to provide 
abundant bandwidth on demand, at any time and from anywhere, to promote bandwidth-rich networked applications. In addition, other government-funded R\&D projects relevant to the photonic networks, including photonic devices and subsystems and large-scale optical interconnect for UHPC have also been described.

Acknowledgments. The author would like to thank S.Araki of NEC, O.Ishida of NTT Labs., T.Morioka of NICT, Y.Nakano of Univ. Tokyo, H.Onaka of Fujitsu, and A.Takada of NTT Labs. for providing materials for the manuscript.

\section{References}

1. K. Kitayama, T. Miki, T. Morioka, H. Tsushima, M. Koga, K. Mori, S. Araki, K. Sato, H. Onaka, S. Namiki, and T. Aoyama, "Photonic network R\&D activities in Japan - Current activities and future perspectives," IEEE J. Lightwave Technol., Vol.23, No.10, pp.3404-3418, 2005.

2. http://www.jgn.nict.go.jp/

3. N.Kataoka, N.Wada, F.Kubota, K.Sone, Y. Aoki, H.Miyata, H.Onaka, and K. Kitayama, "Field trial of data-granularity-flexible reconfigurable OADM with wavelength-packet-selective switch," IEEE J. Lightwave Technol., vol.24, No.1, pp.88-94, 2006.

4. T.Hasegawa, T.Nishioka, H.Ishizuka, J.Abe, K.Shimizu, and M.Matsui, "Field experiments of quantum cryptosystem in 96km installed fibers," CLEO/EuropeEQEC, EH3-4-WED, Munich, 2005.

5. A. Al Amin, K. Shimizu, M. Takenaka, T. Tanemura, R. Inohara, K. Nishimura, Y. Horiuchi, M. Usami, Y. Takita, Y. Kai, Y. Aoki, H. Onaka, Y. Miyazaki, T. Miyahara, T. Hatta, K. Motoshima, T. Kagimoto, T. Kurobe, A. Kasukawa, H. Arimoto, S. Tsuji, H. Uetsuka and Y. Nakano, "40/10Gbps bit-rate transparent burst switching and contention resolving wavelength conversion in an optical router prototype," ECOC2006, Th.4.1.6 (Cannes, Sep.2006).

6. http://www.psi-project.jp/

7. H.Onaka, Y.Aoki, K.Sone, G.Nakagawa, Y.Kai, S.Yoshida, Y.Takita, K.Morito, S.Tanaka, and S.Kinoshita, "WDM Optical Packet Interconnection using MultiGate SOA Switch Architecture for Peta-Flops Ultra-High-Performance Computing Systems,” ECOC2006 Tu4.6.6(Cannes, Sep.2006). 